\title{
Cosmology of Spin-2 Fields
}

\author{
M. D. Maia \\ University of Brasilia, Institute of Physics \\ 70910-970, Brasilia DF \\ maia@unb.br
}

June 7, 2021

\begin{abstract}
The Cauchy-Kowalevski theorem is applied to the solutions of Einstein's equations and to cosmology. Three fundamental requirements of the theorem: the use of analytic series; the existence of the boundary surfaces; and the setting of the independent initial data are revised, using methods of geometric analysis. It is shown that during its relativistic phase, the standard model of the universe is governed by Einstein's gravitation described as a massless spin-2 field, but it is necessarily complemented by massive spin-2 field, which responds for the dark sector of the universe. On the other hand, at the inflationary phase, the exponential growth of the volume of the universe is shown to be consistent with a thermal, non-relativistic expansion. These two phases are separated by the last inflationary surface.
\end{abstract}

\section{Gravitation and Spin-2 Fields}

The standard model of the universe describes a causal sequence of space-like 3dimensional surfaces of the space-time manifold (for short, these will be referred to simply as surfaces) evolving along a global time-like line. Current observations tell that these surfaces are flat in the Riemann sense. Furthermore, the observations of supernovae type Ia show that these surfaces are subjected to a non-vanishing tension, resulting from the unbalance between ordinary gravitating matter and some yet unknown dark force, producing an acceleration in the universe expansion. The purpose of this note is to show that these observations are consistent with the proper formulation of the Cauchy problem in General Relativity. 
The spin-statistics theorem of relativistic quantum mechanics show that quantum systems with integer spin obey the Einstein-Bose statistics and those with half integer spins obey the Fermi-Dirac statistics. One of its consequences is that Einstein's gravitation fits the Einstein-Bose statistics as a massless spin-2 field. The relation between the spin and the classical degrees of freedom of a field $D_{f}$ measured in the 3-dimensional space-like hypersurfaces of the space-time is $D_{f}=(2 s+1)$. Thus, scalar, vector and symmetric tensor field are related to spin-0, spin-1 and spin-2 quantum fields respectively [1, 2].

In compliance with the spin-statistics theorem, the Lagrangian for a generic spin-2 field defined by a symmetric, non-singular tensor $H_{\mu \nu}$ was described by M. Fierz and W. Pauli in the Minkowski space-time as

$$
\mathcal{L}=\frac{1}{4}\left[H_{, \mu} H^{, \mu}-H_{\nu \rho, \mu} H^{\nu \rho, \mu}-2 H_{\mu \nu}{ }^{, \mu} H^{, \nu}+2 H_{\nu \rho, \mu} H^{\mu \rho, \nu}-m^{2}\left(H_{\mu \nu} H^{\mu \nu}-H^{2}\right)\right],
$$

where $H=\eta^{\mu \nu} H_{\mu \nu}$ and $m$ is the rest mass of the field [3]. Notice that in a 3dimensional surface $H_{\mu \nu}$ would have six degrees of freedom, but one of them is eliminated on the account that the state of smallest energy of the field occurs when 1 $\eta^{\mu \nu} H_{\mu \nu}=H_{i}{ }^{i}+\eta^{44} H_{44}=0$.

The relation between spin-2 fields and gravitation was first noted by S. N. Gupta in 1954, observing that in the case $m=0$, the Euler-Lagrange equations derived from (11) coincide with Einstein's gravitational wave equations obtained from the linear perturbation $g_{\mu \nu}=\eta_{\mu \nu}+\gamma_{\mu \nu}$ of the Minkowski metric $\eta_{\mu \nu}$ (using the de Donder coordinate gauge)

$$
\square^{2} \psi_{\mu \nu}=0, \quad \psi_{\mu \nu}=\gamma_{\mu \nu}-\frac{1}{2} \gamma \eta_{\mu \nu}
$$

In the sequence, Gupta reversed the linear approximation procedure, by adding successive perturbation terms to the Minkowski metric

$$
g_{\mu \nu}=\eta_{\mu \nu}+\gamma_{\mu \nu}+\gamma_{\mu \nu}^{2} \ldots
$$

while at the same time calculating the respective curvature tensors. Comparing the result with the corresponding perturbations of the energy-momentum tensor of the source term, at the end Gupta recovered the full non-linear Einstein's equations

$$
R_{\mu \nu}-\frac{1}{2} R g_{\mu \nu}=8 \pi G T_{\mu \nu}
$$

Here $g_{\mu \nu}$ is regarded as a field on the Minkowski's space-time [4]. Thus, the tensor $R_{\mu \nu}$ and the scalar $R$ in those equations are formally defined as the Ricci tensor and Ricci scalar respectively calculated from $g_{\mu \nu}$ as in Riemannian geometry. Therefore, equations (4) describe a non-linear spin-2, massless field in Minkowski's space-time, which is completely equivalent to the Euler-Lagrange equations derived from the Einstein-Hilbert action $\mathcal{L}=R \sqrt{-g}$ in General Relativity, up to an additional divergence-free term. In order to obtain the full theory of General Relativity it becomes also necessary to identify the gravitational potential $g_{\mu \nu}$ with the spacetime metric geometry, and to postulate the principles of general covariance and of equivalence, which are compatible with Einstein's equations.

The above correspondence is not a coincidence. As it happens with the other fundamental interactions, it provides the missing field theoretic support to Einstein's

\footnotetext{
${ }^{1}$ Our convention: Greek indices run from 1 to 4 and Latin indices run from 1 to 3 .
} 
dynamical equations, which were otherwise derived by pure geometrical considerations [5, 6, 7].

An immediate consequence of the spin-2 characteristics of gravitation is that any alternative relativistic gravitational theory where the gravitational potential is defined by the space-time metric will be necessarily defined by the Einstein-Hilbert action. For example, in the so called $F(R)$ alternative theories of gravitation, where $F$ is a function of the Ricci scalar $R$ derived from the metric tensor taken as the gravitational field, will necessarily lead to $F(R)=R \pm$ (a divergence-free term). To be sure, it is necessary to verify that any other spin-2 field described by (1) does not lead to a valid alternative theory of gravitation. This was checked in the 70's [8, 9], by repeating the same non-linear reconstruction introduced by Gupta, but using the Fierz-Pauli Lagrangian (1) with $m \neq 0$. It was soon found that such theory meets strong disagreements with the classic gravitational experiments, resulting from the non-linear interference of the mass term in the resulting equations. In addition, as a field theory in Minkowski's space-time, it contains tachionic and acausal solutions [10, 11, 12, 13, 14].

It should be added that the proposed "massive gravity" cannot have Einstein's gravity as its zero mass limit. This follows from the fact that in any Poincaré invariant field theory, the concept of mass is given by the eigenvalues of the Casimir mass operator, whose spectrum is composed of a distribution of discrete isolated points in the real line [15]. Such spectrum does not contain an infinite number of arbitrarily small masses, required to define the zero mass limit. Consequently, the massless, non-linear spin-2 field (or particle) in Minkowski space defined by FierzPauli is isolated and independent from the massive Fierz-Pauli spin-2 field. In the following we give a geometrical interpretation for this massive field.

\section{The Cauchy Problem in General Relativity}

The global hyperbolicity of Einstein's equations implies that its causal solutions can be uniquely expressed by a Cauchy sequence of surfaces, orthogonal to an integrable time-like vector field, satisfying the conditions of the Cauchy-Kowalevski theorem. By an integrable vector field it is meant that through the points of each surface passes a curve whose velocity vector is the said vector field. The standard model of cosmology provides a prime example of the solutions of Einstein's equations described by such Cauchy sequence, composed by flat space-like surfaces evolving in a cosmic time.

However, the conditions established by the Cauchy-Kowalevski theorem can be quite restrictive for high energy physics and to General Relativity in particular. The three most important are:

(a) Since all surfaces of a space-time are submanifolds, their metric geometry are necessarily induced by the space-time-metric [16]. Therefore the geometry of the Cauchy surfaces, can be defined only after the problem is solved.

(b) In General Relativity all gravitational properties are derived from the metric, assumed to be the only independent variable. Consequently, the velocity of propagation of the surfaces, which is to be set as one of the initial values, is also dependent of the metric. For example, using coordinates co-moving with these surfaces, the velocity of propagation of the surfaces is expressed by the metric affine connection as

$$
\dot{g}_{i j}=-2 g_{4 \mu} \Gamma_{i j}^{\mu}
$$


which is entirely dependent of the metric and its use to build the Cauchy problem, can lead erroneous conclusions.

(c) Finally, the standard proof of the Cauchy-Kowalevski theorem holds for analytic functions of the coordinates. In General Relativity and in most non-linear differential equations of physics the best we may hope is to use differentiable functions. This problem is actually a major issue in the analysis of Riemannian manifolds, which has been solved only recently with the development of "Geometric Analysis": The solutions of non-linear differential equations by use of "smooth deformations of surfaces". Due to the relevance of this concept to General Relativity, we end this section with a brief introduction, while referring the details to the original cited sources.

We find it more intuitive to start with the proof of the Poincaré Conjecture, which shows how to continuously deform a closed compact surface into a sphere. The proof uses thermodynamics to determine the variation of the volume enclosed by a compact surface with the temperature. Assuming that the volume vary exponentially with the temperature (or with the entropy) $u, \int \sqrt{g} d v=\int e^{u} d v$. Replacing $\sqrt{g}=e^{u}$ in Fourier's heat equation, it follows that $\frac{\partial u}{\partial t}=\frac{1}{2} g^{\mu \nu} \frac{\partial g_{\mu \nu}}{\partial t}$. Comparing this result with the expression of the Ricci tensor, written in geodesic coordinates, after canceling the trace, we find the Ricci flow expression

$$
\dot{g}_{i j}=-2 R_{\mu \nu}
$$

which specifies the velocity of the "deformation of the geometry" by the Ricci tensor[17]. In 2002, G. Perelmann applied (6) to prove the Poincaré conjecture [18]. Using an intuitive approach, this can be seen in the following way: Consider a sphere $\Sigma$ immersed in a 3 -dimensional compact surface $\bar{\Sigma}$, near a heat source. Next, mark the positions of the heat flow lines and smoothly push the surface $\bar{\Sigma}$, so that it always remains orthogonal to the heat flow lines, without creating wrinkles, until it coincides with the enclosed sphere.

It can be easily seen that Perelmann's result cannot be applied to General Relativity. Indeed, by replacing (6) in Einstein's equations it implies in a linear gravitation and in the vacuum, gravitation would not propagate at all. However, a similar but more general concept of smooth deformation of surfaces was developed in 1956 by John Nash, applicable to any Riemannian manifold. Nash's original motivation was to prove the existence of submanifolds of a Riemannian manifold by use of differentiable functions only [19]. Before Nash, the only known solutions of the Gauss-Codazzi equations, which determines the existence of surfaces in a manifold, requires the use of analytic series. Nash's theorem solved this problem by applying a new concept of smoothing operators, similar but more general than (5) or (6) , using the extrinsic curvature of the surface. The extrinsic curvature gives a local measure of how a surface deviates locally from its tangent plane. It is therefore a measure of the local curvature of a surface which is not entirely dependent of the surface metric.

The Nash flow expression can be derived from the propagation of an object $\bar{\Omega}$ defined in one surface $\bar{\Sigma}$, when it is Lie transported to another surface. That is, along the integral curve of the orthogonal vector field $\eta: \Omega=\bar{\Omega}+t\left(£_{\eta} \Omega\right)$, where $t$ is the parameter of the curve. In particular, taking $\bar{\Omega}$ to be the metric $\bar{g}_{i j}$ and $\bar{k}_{i j}$ of the initial surface, we obtain in the new surface the polynomial expansions [20, 21]

$$
\begin{aligned}
g_{i j}(x, t) & =\bar{g}_{i j}-2 t \bar{k}_{i j}+t^{2} \bar{g}^{m n} \bar{k}_{i m} \bar{k}_{j n} \\
k_{i j}(x, t) & =\bar{k}_{i j}-t \bar{g}^{m n} \bar{k}_{i m} \bar{k}_{j n} .
\end{aligned}
$$


Comparing the derivative of the first expression with the second expression we easily obtain the Nash Flow2

$$
\dot{g}_{i j}=-2 k_{i j}
$$

\section{Relativistic Cosmology}

In the application to the Cauchy-Kowalevski theorem in General Relativity, the condition (7) provides the velocity propagation of the surface geometry in terms of a quantity which is independent of the metric and of coordinate choices. Since $k_{i j}$ is a rank-2 tensor, then it also corresponds to a spin-2 field, defined by the Fierz-Pauli dynamics. However, we have seen that such dynamics always leads to Einstein's equations when the mass $m$ in (1) is zero, so that $k_{i j}$ must correspond to a massive spin-2 field defined by the Fierz-Pauli Lagrangian defined in a 3-dimensional surface of space-time, obtained from (11) with $H_{i j}=k_{i j}(x, t), H_{i 4}=0$ and $H_{44}=h(x, t)$, where $h(x, t)$ is the mean curvature of the surface

$$
\mathcal{L}^{m F P}=\frac{1}{4}\left[h_{, i} h^{, j}-k_{j k ; i} k^{j k ; i}-2 k_{i j}{ }^{; i} h^{, j}+2 k_{j k ; i} k^{i k ; j}-m^{2}\left(K^{2}-h^{2}\right)\right] \sqrt{-g} .
$$

Here $K^{2}=k^{i j} k_{i j}$ is the Gaussian Curvature of the surfaces and their mean curvature of are defined by $h=g^{i j} k_{i j}$. The indicated covariant derivatives are calculated with the metric $g_{\mu \nu}$ of the space-time and its surface- induced metric and connection.

The field equations for $k_{i j}$ are

$$
\left(\square^{2}-m^{2}\right) \Psi_{i j}=0, \quad \Psi_{i j}=k_{i j}-h g_{i j},
$$

where $\square^{2}=g^{i j} \nabla_{i} \nabla_{j}$, and $\Psi_{i j}$ may be called the deformation wave function so as not to be confused with massive gravity.

Since the dynamics of $k_{i j}$ is independent of the metric, the field $k_{i j}$ does not enter in the geometric side of Einstein's equations but it contributes as a source field through its energy tensor, derived from the potential energy of (8):

$$
T_{i j}^{m F P}=\frac{m^{2}}{2}\left(k_{i}^{m} k_{m j}-h k_{i j}-\frac{1}{4}\left(K^{2}-h^{2}\right) g_{i j}\right) .
$$

In cosmology the line element of the Friedmann, Lemaitre, Robertson, Walker (FLRW) standard model, written in a coordinate system which is co-moving with the Cauchy surfaces, can be written as

$$
d s^{2}=-d t^{2}+a(t)^{2}\left(d r^{2}+f^{2}(r) r^{2} d \omega^{2}\right),
$$

where $f(r)=(\sinh r, r, \sin r)$, corresponding respectively to open, flat and closed universes (equivalently, for $d r \rightarrow \frac{d r}{1-\kappa r^{2}}$, with $\kappa=(-1,0,1)$ ). Replacing the FLRW metric components in Einstein's equations and considering only the perfect fluid as a source we obtain the standard Friedmann's equation for

$$
\dot{a}^{2}+\kappa=\frac{8 \pi G}{3} \rho a^{2} .
$$

Notice that the standard derivation of Friedmann's equation does not include an initial surface and an initial data required by the Cauchy-Kowalevski theorem. Any

\footnotetext{
${ }^{2}$ In the ADM formulation of General Relativity the same condition (7) is known as the York relation [22].
} 
attempt to add such data at this stage by using (5) or (6) would make the propagation velocity entirely dependent of the metric and consequently, it may induce errors. In the following we start anew, regarding the FLRW cosmology, as a Cauchy problem with the use of (7).

Assuming that the inflation occurred isotropically, the Last Inflationary Surface (LIS for short), just before as the universe entered the FLRW period, would be a 3-sphere, which is therefore taken as the initial surface for the relativistic universe. An spherical initial surface naturally leads to Legendre polynomials, which form an orthogonal and complete Fourier basis), independently of further boundary conditions.

With the presence of the energy-momentum tensor in the right hand side of Einstein's equations we obtain

$$
R_{\mu \nu}=-8 p i G\left(T_{\mu \nu}-\frac{1}{2} T g_{\mu \nu}\right)+T_{\mu \nu}^{m}-\frac{1}{2} T^{m} g_{\mu \nu}
$$

These equations can be solved for the FLRW metric in the same way the original derivation of Friedmann's equation. The difference is that here we have a Cauchy problem with initial value for $k_{i j}$ at the LIS specifying that $\left.\left.k_{i j}\right\rfloor_{L I S} \propto g_{i j}\right\rfloor_{L I S}$, which implies that the LIS is a constant curvature surface [16.

After the LIS, the observations show that the Cauchy surfaces are flat. Therefore, they must result from a continuous deformation from the LIS, generating a sequence of flat or nearly flat surfaces. This may be obtained with the supposition that $k_{i j}=\alpha(t) g_{i j}$ (the proportionality factor is a function of time). More specifically, for the FLRW metric we may write

$$
k_{i j}=\frac{b(t)}{a^{2}(t)} g_{i j} .
$$

where $b(t)=k_{11}$ remains an arbitrary function, representing the radial tension on the surface. With this notation, the energy tensor resulting from (10) for the FLRW universe becomes

$$
T_{i j}^{m F P}=\frac{m^{2}}{4} \frac{b^{2}}{a^{2}} g_{i j}
$$

It is more convenient to define the cosmological function $\Lambda(t)$ by

$$
\frac{m^{2}}{4} \frac{b^{2}}{a^{2}} \stackrel{\text { def }}{=} \frac{\Lambda(t)}{3}
$$

so that after replacing in Einstein's equations we obtain the modified Friedmann's equation

$$
\dot{a}^{2}+\kappa=\frac{8 \pi G}{3} \rho a^{2}-\frac{\Lambda(t)}{3} a^{2} .
$$

The value of $\Lambda(t)$ can now be determined by the observed acceleration of the universe, which is indicated by the observations and that fits the $\Lambda$ CDM paradigm as a cosmological constant.

$$
\Lambda(t)\rfloor_{\text {observed }} \approx 10^{-47} \mathrm{Gev}^{4} \sim 10^{-29} \mathrm{~g} / \mathrm{cm}^{3} .
$$

Replacing this value in (15) and using the same $\Lambda$ CDM paradigm, we may determine the mass $m$ from the estimates for dark matter. For example using the Weakly Interacting Massive Particle (WIMP) dark matter model the value of $m$ would be $m \approx 100 \mathrm{Gev}$. Using this mass in (15), the component $k_{11}(=b(t))$ may be determined. Therefore all components of the Cauchy problem for the present relativistic phase of the universe are determined. 


\section{A Distant Mirror}

The existence of an inflationary phase of the universe was proposed to correct the predicted age of the FLRW universe as compared with the observed age of some stars. The supposition was that during the period of $\approx 10^{-32}$ seconds, the volume of the universe increased exponentially, something very unlike the slow expansion observed today. As we have seen, one such rapid growth of volume can be explained by an exponential function of the entropy. In particular for $\sqrt{g}=e^{u}$, we obtain Ricci flow deformation given by (6). Since this is not compatible with Einstein's equations used in the relativistic phase, we are in presence of two distinct geometries, with a proper junction condition between them at the LIS.

It is possible that the inflationary phase can be explained as a Cauchy problem, by establishing an initial surface, say at the Big Bang horizon, regarded as a compact and closed surface into which all time-like geodesics converge. Assuming that the expansion of the inflationary universe is regulated by the Ricci flow (6), then by a similar demonstration of the Poincaré conjecture, the Big Bang horizon, deforms into the LIS spherical surface. In that case, the LIS would be a junction between a geometry defined by $R_{i j}$ and another defined by Einstein's equations and the Nash flow (7), that is by $k_{i j}$.

The Israel-Lanczos junction condition is compatible with both thermal and relativistic dynamics, because it compares the behavior of the tangent and normal components of the two geometries when passing from one side of the LIS to the other [23]. To understand this note that the inflationary side of LIS, the Ricci curvature of the surface is defined only by the variations of the tangent vectors, so that there is no change. However, in the relativistic side the extrinsic curvature depends on the variation of the normal vector to the surface, which varies from one side to another. Therefore the junction condition at the LIS requires that

$$
\left.\left.R_{i j}\right\rfloor_{\text {LIS }}=k_{i j}\right\rfloor_{\text {LIS }}
$$

This condition is compatible with Einstein's equations, only if we impose that the surface has a $Z_{2}$ symmetry, or more intuitively if it acts as a mirror, leading to the expression known as the Israel-Lanczos condition (For a detailed derivation see [24])

$$
k_{i j}=-8 \pi G\left(T_{i j}^{m F P}-\frac{1}{3} T^{m F P} g_{i j}\right)
$$

which gives the extrinsic curvature of the LIS in terms of the energy tensor of the then existing gravitational source, the massive spin-2 field. Since the inflation happened before the structure formation of ordinary matter and even before the radiation condensation period of the universe, atoms and gauge forces cannot compose that energy-momentum tensor in (18). The only remaining possibility is that such energy-momentum tensor results from the existence of the massive Fierz-Pauli spin-2 field given by (10).

The existence of such massive spin-2 field can be explained by today's observation of the acceleration of the universe and the lack of mass galaxies.

The existence of a massive spin-2 field acting as a dark matter content may justify the additional gravitational pull, stopping the currently observed acceleration, thus avoiding the big rip scenario for end of the universe. Quite on the contrary, the present universe may follow the arguments similar to an "inverse Poincaré conjecture", whereby the compact and closed LIS can smoothly deform into another 
compact closed surface (and never into an open universe), because a closed compact space cannot be smoothly deformed into an open volume. Clearly, this requires a relativistic generalization of the proof of the Poincaré conjecture using the Nash flow instead of the Ricci flow.

In conclusion, the Cauchy-Kowalevski theorem was reviewed and applied to General Relativity and cosmology, using two new tools: The "geometrical analysis" which eliminates the necessity to use of analytic functions by the use of the concept of geometric flow. More importantly it leads us to the use of extrinsic curvature as a massive spin-2 field, required to complete the set of independent initial data required by the theorem. In the beginning, the universe was described as being predominantly thermal, promoting an exponential growth of the volume of the space-time with the temperature, with the Ricci-flow geometry. This was followed by today's standard relativistic universe, which is compatible with Nash flow geometry. The free parameters were adjusted by the available observational data within the $\Lambda \mathrm{CDM}$ paradigm. The additional massive spin-2 field required by the Cauchy-Kowalevski theorem is also regarded as a possible candidate to dark matter, while its energy tensor contributes to dark energy. The Last Inflationary Surface (LIS) joins the thermal and relativistic phases of the universe, acting as a boundary with a mirror symmetry. The existence of such symmetry suggests that the present universe can be regarded as a reflection of a past universe

\section{Acknowledgements:}

The author wishes to acknowledge professor Stanley Deser for comments and suggestions on a earlier version of this paper. 


\section{References}

[1] M. Fierz, Helvetica Physica Acta 12, 3 (1939).

[2] W. Pauli, Phys. Rev. 58, 716 (1940).

[3] M. Fierz and W. Pauli, Proc. Roy. Soc. Lond. A 173, 211 (1939).

[4] S. N. Gupta, Phys. Rev. 96, 1683 (1954).

[5] R. P. Feynman, Fernando B. Morino and William G. Wagner Lectures on Gravitation. Addison Wesley Publishing company. Menlo Park, Ca, USA.

See the Foreword by John Preskill and Kip S. Thorn. (1962).

[6] C. Fronsdal, Phys. Rev. D 18, 3624 (1978).

[7] C. Sivaram and K.P. Sinha, Phys. Rep, 51, 111, (1979).

[8] H. Van Dam and M. J. Veltman, Nucl. Phys. B 22, 397 (1970).

[9] V. Zakharov, JETP Lett. 12, 312, (1970).

[10] D. G. Boulware and S. Deser, Phys. Rev. D 8, 3368 (1972).

[11] S. Deser and M. Henneaux, Class. Quantum Grav. 24, 1683 (2007)

[12] S. Deser, K. Izumi, Y.C. Ong, A.Waldron, Massive Gravity Acausality Redux, arXiv:1306.5457 (2013).

[13] Kurt Hinterbichler, Rev. Mod. Phys. 84, 671 (2012)

[14] Claudia de Rham, Massive Gravity, www.livingreviews.org/lrr-2014-7 (2014).

[15] M. Flato and D. Sternheimer, Phys. Rev. Lett. 15, 934 (1965).

[16] L.P. Eisenhart, Riemannian Geometry, Pinceton U. P. sixth print (1966).

[17] R. S. Hamilton, J. Diff. Geom. 17, 255 (1982).

[18] G. Perelmann, The Entropy Formula for the Ricci Flow and its Geometric Applications, ArXiv:math/0211159 (2002).

[19] J. F. Nash. Annals of Maths. 63, 20 (1956)

[20] M. D. Maia, Phys. Rev. D 31, 262 (1985); M. D. Maia, Phys. Rev. D 31, 268 (1985).

[21] M. Crampin and F. A. E. Pirani, Applicable Differential Geometry, Cambridge U. Press, U.K. (1986).

[22] Y. Choquet-Bruhat and J. York Jr. Mathematics of Gravitation, Institute of Mathematics, Polish Academy of Sciences, Warsaw (1997). Also, J. M. York Jr. The Initial Value Problem Using Metric and Extrinsic Curvature arXiv:gr-qc/0405005v1 (2004).

[23] W. Israel, Il Nuovo Cimento, 44, 4349, (1966).

[24] M. D. Maia, E. M. Monte, J. M. F. Maia and J. S. Alcaniz. Class. Quantum Grav.22, 1623, (2005). doi:10.1088/0264-9381/22/9/010. 